\title{
SASKATCHEWAN CHRISTMAS MAMMAL COUNT - 1985
}

Compiled by WAYNE C. HARRIS, Box 414, Raymore, Saskatchewan. SOA 3J0

A total of 62 counts were received this year reporting 32 species during the count period. The number of counts exceeds the previous high of 55 received in 1983 while the total number of species is two less than the record high reported last year.

There were no new species reported this year. There were, however, several rare species reported. Fox Squirrels were again found at Regina and Weyburn, the latter having seven seen and eight nests found. Both Fisher and River Otter were seen at Prince Albert National Park and a Richardson's Ground Squirrel was found at Moose Jaw. The report of Timber Wolves near Indian Head is well south of the normal range of this species.

From a population viewpoint Mink seem to have recovered from last year's low and were the only species to show a significant increase over last year. Far more species showed decreases including White-tailed Jack Rabbit (down from last year's already decreased populations), Porcupine, White-tailed Deer and Pronghorn. The last two may very well be a result of the severe winter last year, however no plausible explanation can be found for the other two. The White-tailed Deer populations were most notably down in central Saskatchewan and holding their own in the southwest as were Mule [eer, of which 182 individuals were seen on the Fort Walsh count. Very encouraging was the report of 11 Moose at Loon Lake where Moose populations have been quite low for close to 10 years.
For weather, coverage and participants on these counts please refer to the Christmas Bird Counts found in this issue. Symbols found in the tables are as follows:

* identified by tracks with estimated number of individuals in parentheses

+ seen during count period but not on count day

$L$ active lodges or huts seen with number in parentheses

$N$ nests found with number seen in parentheses

$D$ fresh diggings seen

d freshly dead animals seen

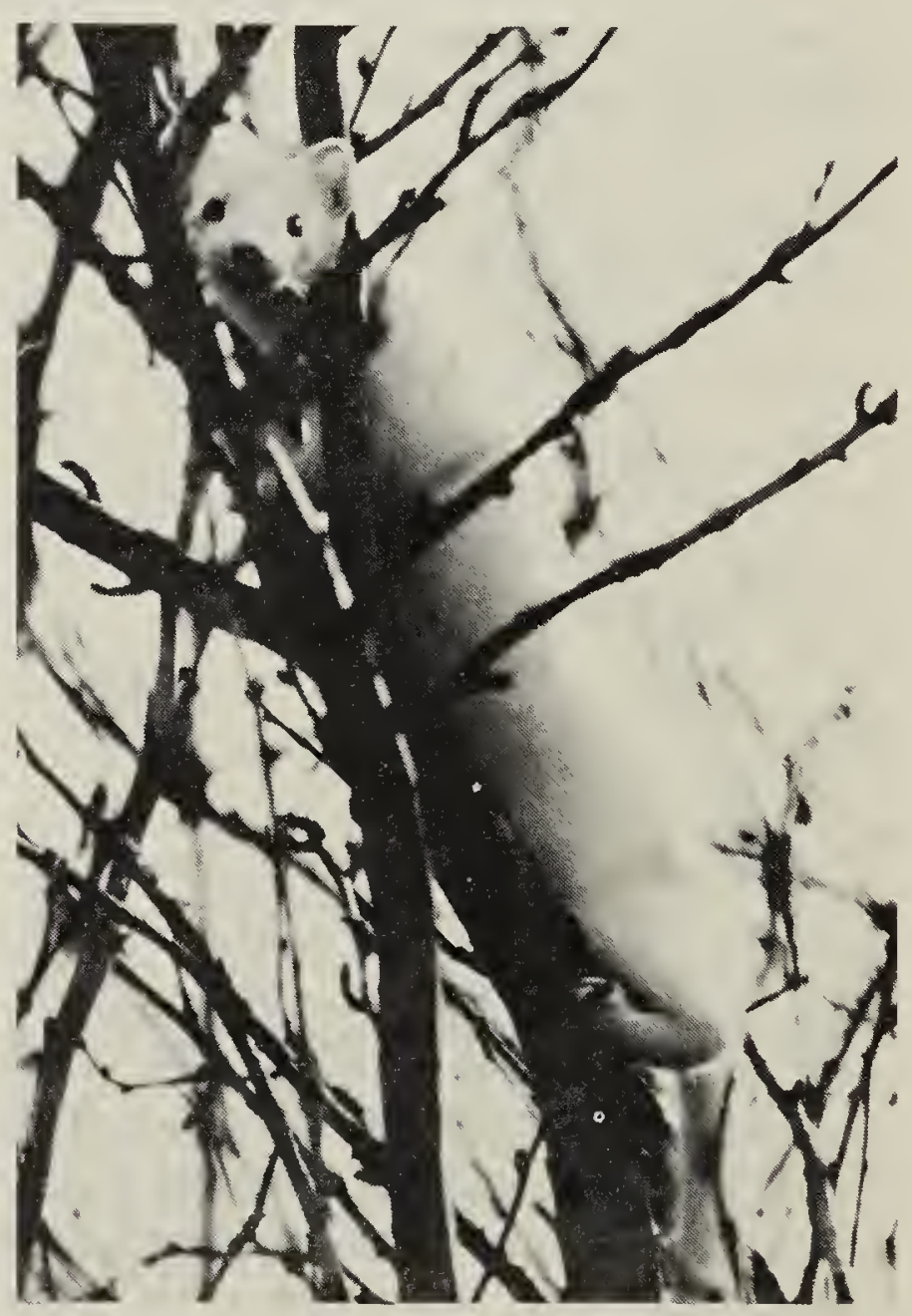

Long-tailed Weasel

Lorne Scott 
Table 1-1. SASKATCHEWAN CHRISTMAS MAMMAL COUNTS

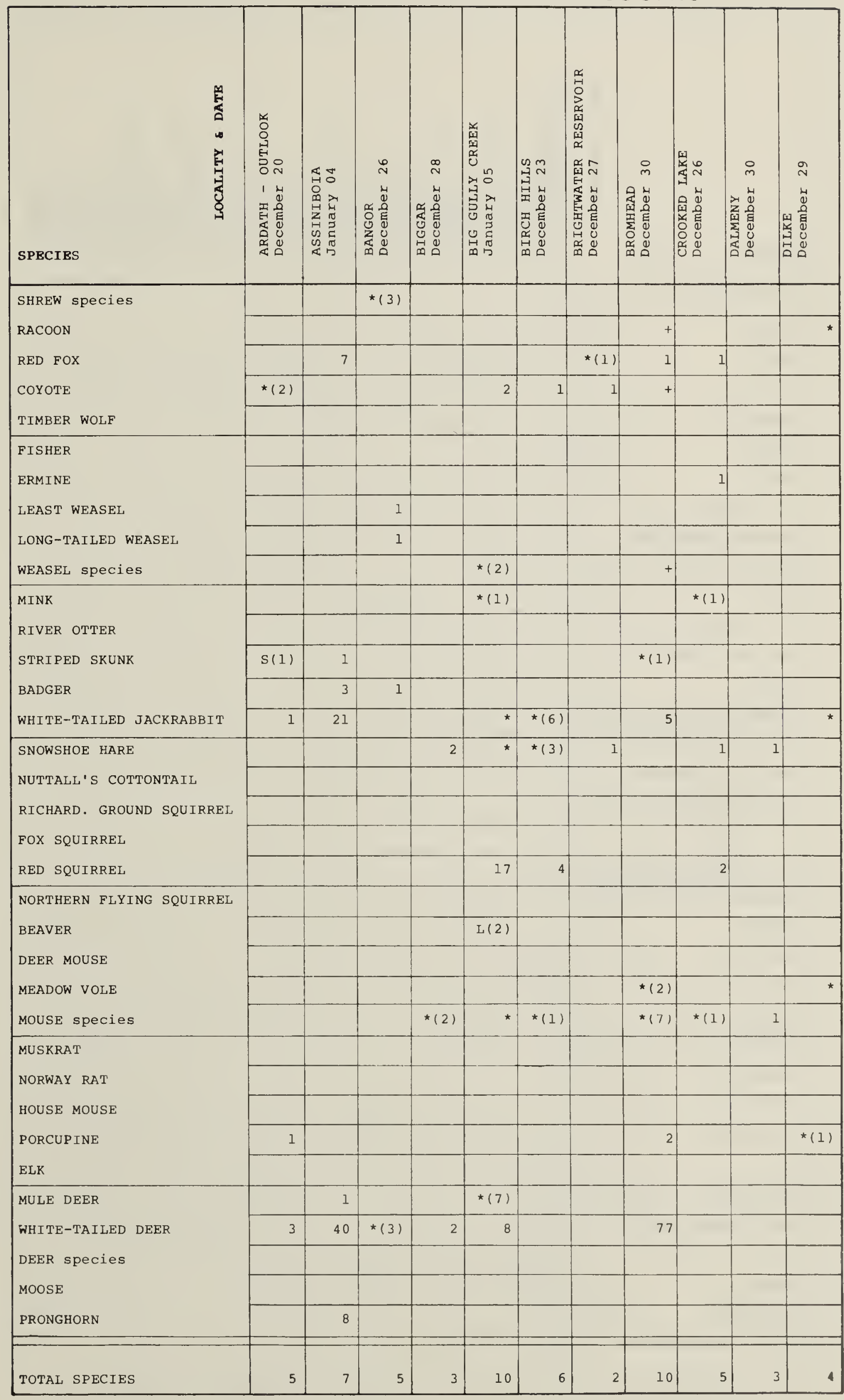


Table 1-2. SASKATCHEWAN CHRISTMAS MAMMAL COUNTS

SPECIES
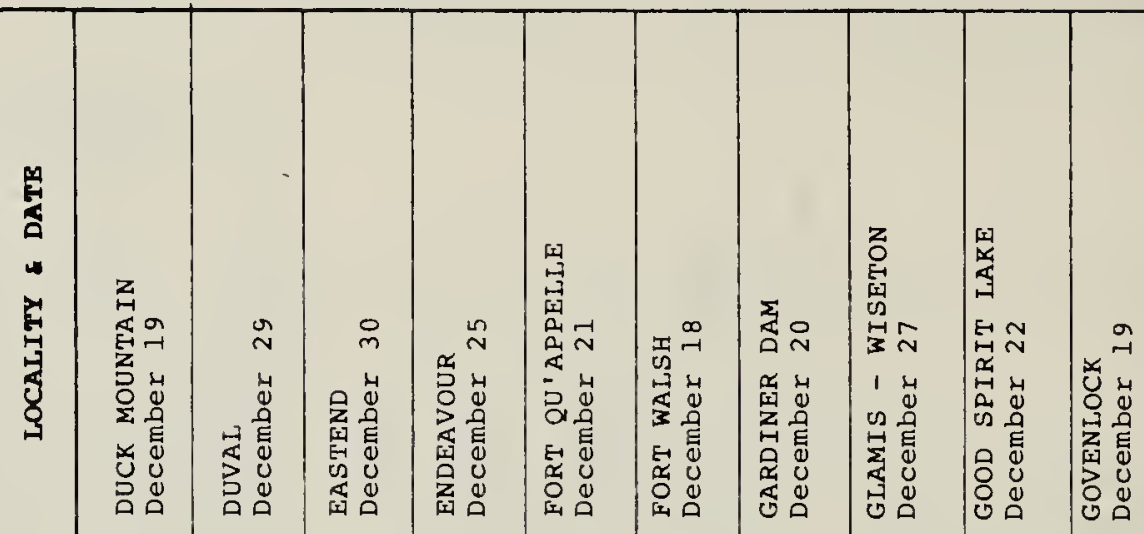

SHREW species

RACOON

RED FOX

COYOTE

TIMBER WOLF

FISHER

ERMINE

LEAST WEASEL

LONG-TAILED WEASEL

WEASEL species

MINK

RIVER OTTER

STRIPED SKUNK

BADGER

WHITE-TAILED JACKRABBIT

SNOWSHOE HARE

NUTTALL'S COTTONTAIL

RICHARD. GROUND SQUIRREL

FOX SQUIRREL

RED SQUIRREL

NORTHERN FLYING SQUIRREI

BEAVER

DEER MOUSE

MEADOW VOLE

MOUSE species

MUSKRAT

NORWAY RAT

HOUSE MOUSE

PORCUP INE

ELK

MULE DEER

WHITE-TAILED DEER

DEER species

MOOSE

PRONGHORN

TOTAL SPECIES

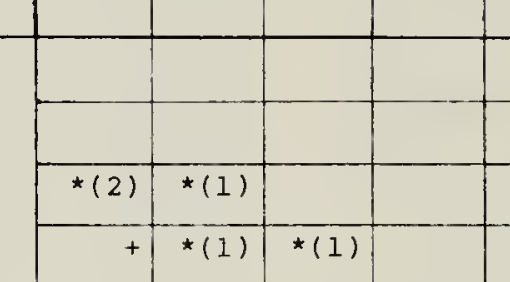

$++$

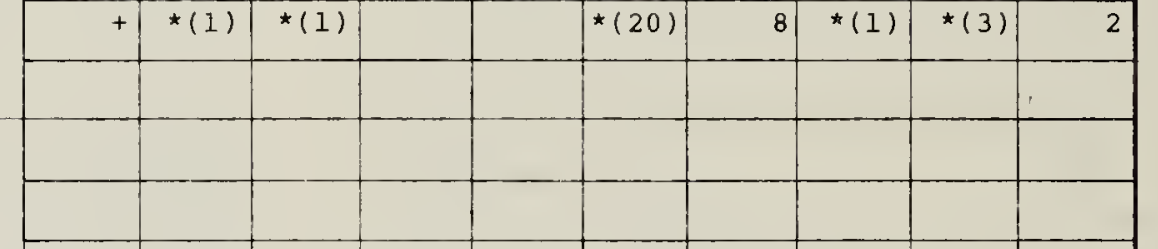

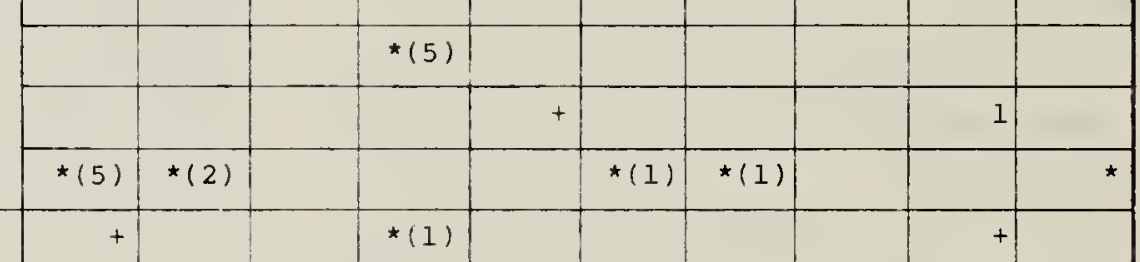

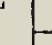

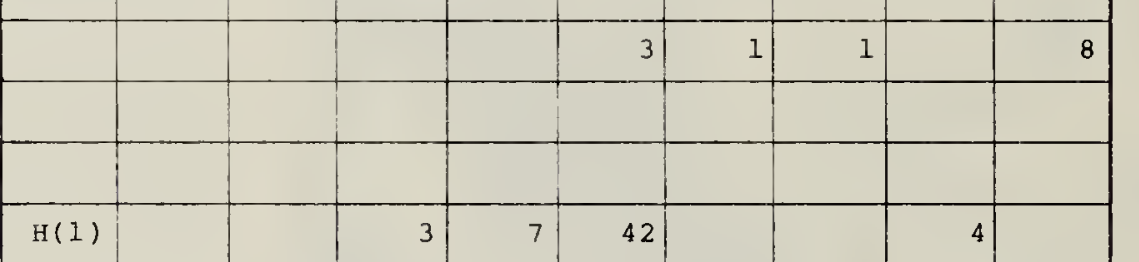


Table 1-3. SASKATCHEWAN CHRISTMAS MAMMAL COUNTS

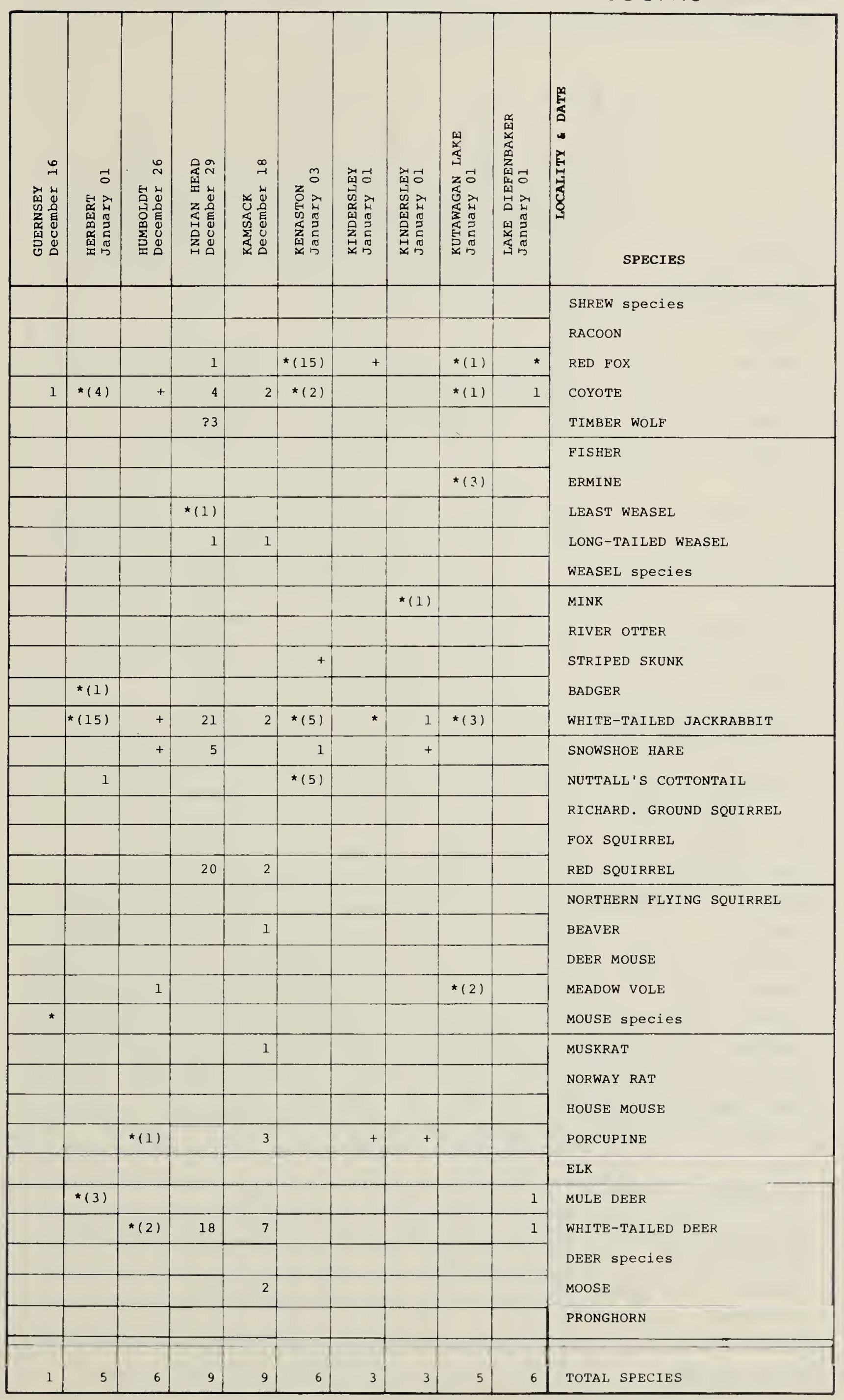


Table 1-4. SASKATCHEWAN CHRISTMAS MAMMAL COUNTS

\begin{tabular}{|c|c|c|c|c|c|c|c|c|c|c|}
\hline 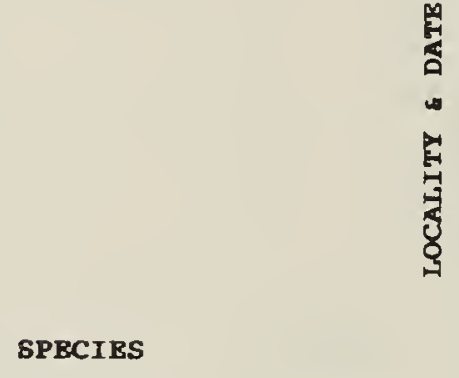 & 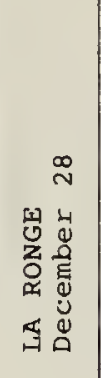 & 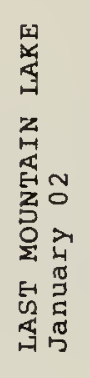 & 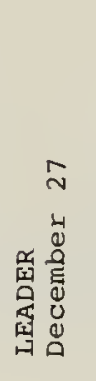 & 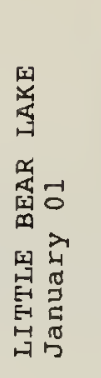 & 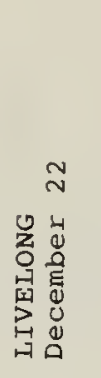 & 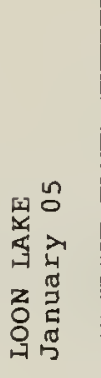 & 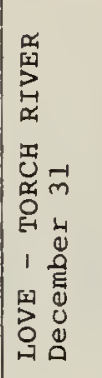 & 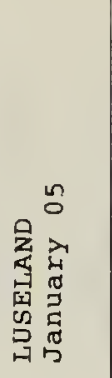 & 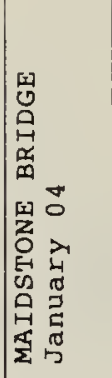 & 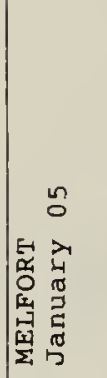 \\
\hline \multicolumn{11}{|l|}{ SHREW species } \\
\hline \multicolumn{11}{|l|}{ RACOON } \\
\hline \multirow{3}{*}{$\begin{array}{l}\text { RED FOX } \\
\text { COYOTE } \\
\text { TIMBER WOLF }\end{array}$} & $\star(2)$ & $\star(1)$ & & & & & $\star(1)$ & 1 & $\star(1)$ & \\
\hline & $\star(1)$ & 2 & $H(1)$ & *(1) & & 1 & *(1) & $\star(4)$ & 3 & \\
\hline & & & & & & & $\star(1)$ & & & 1 \\
\hline \multicolumn{11}{|l|}{ FISHER } \\
\hline \multirow{4}{*}{$\begin{array}{l}\text { ERMINE } \\
\text { LEAST WEASEL } \\
\text { LONG-TAILED WEASEL } \\
\text { WEASEL SPECIES }\end{array}$} & *(4) & & & & & & & & 1 & \\
\hline & & & & & & & & & & \\
\hline & & & & & & & & & 1 & \\
\hline & & * & & & & & & & *(2) & \\
\hline \multirow{5}{*}{$\begin{array}{l}\text { MINK } \\
\text { RIVER OTTER } \\
\text { STRIPED SKUNK } \\
\text { BADGER } \\
\text { WHITE-TAILED JACKRABBIT }\end{array}$} & $\star(1)$ & & & $\star(1)$ & & & + & & *(1) & \\
\hline & & & & & & & & & & \\
\hline & & & & & & & & & *(1) & \\
\hline & & & & & & & & & & \\
\hline & & 7 & 2 & & 1 & & $\star(2)$ & 3 & *(5) & \\
\hline \multirow{5}{*}{$\begin{array}{l}\text { SNOWSHOE HARE } \\
\text { NUTTALL'S COTTONTAIL } \\
\text { RICHARD. GROUND SQUIRREL } \\
\text { FOX SQUIRREL }\end{array}$} & $\star(6)$ & & & $\star(1)$ & & & & $\star(16)$ & 3 & \\
\hline & & & 2 & & & & & & & \\
\hline & & & & & & & & & & \\
\hline & & & & & & & & & & \\
\hline & 4 & & & & 1 & & 2 & & 2 & 5 \\
\hline \multicolumn{11}{|l|}{ NORTHERN FLYING SQUIRREL } \\
\hline \multirow{4}{*}{$\begin{array}{l}\text { BEAVER } \\
\text { DEER MOUSE } \\
\text { MEADOW VOLE } \\
\text { MOUSE species }\end{array}$} & & & & & & & & & $L(3)$ & \\
\hline & & & & & & & & & & \\
\hline & & & & & & & & & & \\
\hline & $\star(6)$ & & & & & & & $\star(6)$ & & * \\
\hline \multirow{5}{*}{$\begin{array}{l}\text { MUSKRAT } \\
\text { NORWAY RAT } \\
\text { HOUSE MOUSE } \\
\text { PORCUPINE } \\
\text { ELK }\end{array}$} & & * & & & & & & & *(1) & \\
\hline & & & & & & & & & & \\
\hline & & & & & & & & & & \\
\hline & & & & & + & & & $\star(1)$ & & \\
\hline & & & & & & & & & & \\
\hline \multirow[b]{2}{*}{ WHITE-TAILED DEER } & & & 4 & & & 20 & & & 2 & \\
\hline & *(1) & 3 & 10 & & & 24 & 2 & $\star(2)$ & 3 & \\
\hline \multicolumn{11}{|l|}{ DEER species } \\
\hline MOOSE & & & & & & 11 & $\star(2)$ & & & \\
\hline PRONGHORN & & & 22 & & & & & & & \\
\hline & & & & & & & & & & \\
\hline TOTAL SPECIES & 8 & 6 & 6 & 3 & 3 & 4 & 8 & 7 & 13 & 2 \\
\hline
\end{tabular}


Table 1-5. SASKATCHEWAN CHRISTMAS MAMMAL COUNTS

\begin{tabular}{|c|c|c|c|c|c|c|c|c|c|c|}
\hline \multirow[t]{9}{*}{$\begin{array}{ll} & N \\
3 & \\
3 & y \\
5 & 0 \\
9 & a \\
0 & 0 \\
0 & 0 \\
0 & 0 \\
\vdots & 0\end{array}$} & 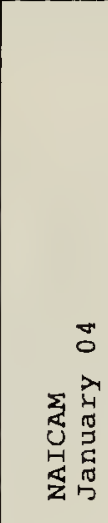 & 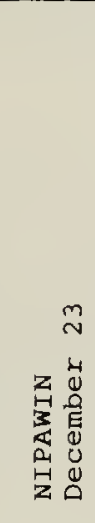 & 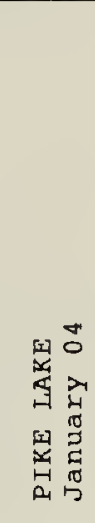 & 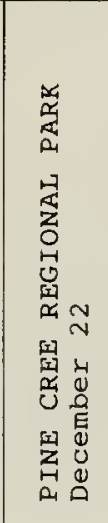 & 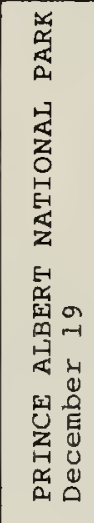 & 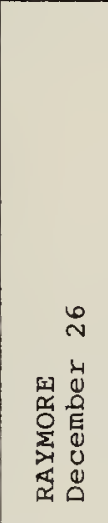 & 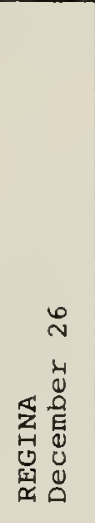 & 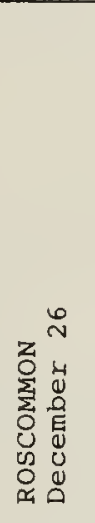 & 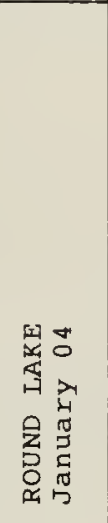 & 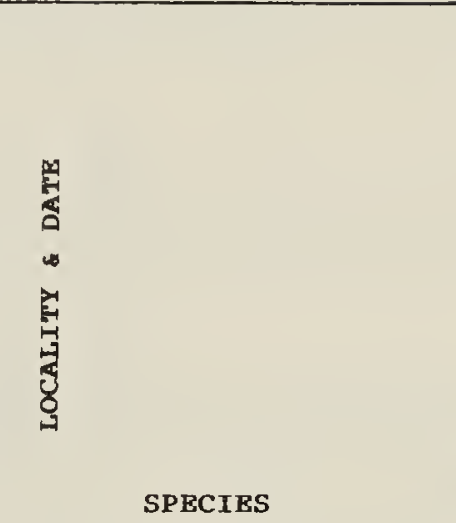 \\
\hline & & & & & & * & & & & \multirow{5}{*}{$\begin{array}{l}\text { SHREW species } \\
\text { RACOON } \\
\text { RED FOX } \\
\text { COYOTE } \\
\text { TIMBER WOLF }\end{array}$} \\
\hline & & & & & & & & & 1 & \\
\hline & 1 & $*(3)$ & $*(1)$ & *(1) & 1 & 1 & 2 & $*(1)$ & & \\
\hline & $\star(2)$ & $*(1)$ & $*(5)$ & & & 2 & $*(1)$ & $*(1)$ & $\star(9)$ & \\
\hline & & & & & $\mathrm{H}(4)$ & & V & & & \\
\hline & & & & & $\star(1)$ & & & & & \multirow{5}{*}{$\begin{array}{l}\text { FISHER } \\
\text { ERMINE } \\
\text { LEAST WEASEL } \\
\text { LONG-TAILED WEASEL } \\
\text { WEASEL SPECIES }\end{array}$} \\
\hline & & & & & & 1 & & & & \\
\hline & & & & & & & & & $\star(6)$ & \\
\hline \multirow[t]{9}{*}{$*(1)$} & & & $\star(2)$ & & & 1 & & & $\star(4)$ & \\
\hline & $*(3)$ & & & $\star(2)$ & *(4) & $*(3)$ & & & & \\
\hline & & & & & & & & & *(3) & \multirow{5}{*}{$\begin{array}{l}\text { MINK } \\
\text { RIVER OTTER } \\
\text { STRIPED SKUNK } \\
\text { BADGER } \\
\text { WHITE-TAILED JACKRABBIT }\end{array}$} \\
\hline & & & & & 3 & & & & & \\
\hline & & & & & & *(1) & $s(1)$ & & & \\
\hline & & & & & & $D(1)$ & & & & \\
\hline & & & $*(2)$ & 1 & & * & * & $*(1)$ & *(1) & \\
\hline & $\star(10)$ & & 2 & $\star(4)$ & & * & 1 & & $*(6)$ & \multirow{5}{*}{$\begin{array}{l}\text { SNOWSHOE HARE } \\
\text { NUTTALL'S COTTONTAIL } \\
\text { RICHARD. GROUND SQU } \\
\text { FOX SQUIRREL } \\
\text { RED SQUIRREL }\end{array}$} \\
\hline & & & & $*(20)$ & & & & & & \\
\hline \multirow[t]{14}{*}{$\star(1)$} & & & & & & & & & & \\
\hline & & & & & & & *(1) & & & \\
\hline & & & 1 & & 2 & 1 & 6 & & 2 & \\
\hline & & & & & & & & & & \multirow{2}{*}{$\begin{array}{l}\text { NORTHERN FLYING SQUIRREL } \\
\text { BEAVER }\end{array}$} \\
\hline & & $*(1)$ & & 1 & & & & & & \\
\hline & 2 & & & & & 1 & & 1 & & \multirow{3}{*}{$\begin{array}{l}\text { DEER MOUSE } \\
\text { MEADOW VOLE } \\
\text { MOUSE species }\end{array}$} \\
\hline & & & & & & 1 & & & & \\
\hline & & & $*(2)$ & $*(8)$ & *(3) & * & $*(1)$ & & *(20) & \\
\hline & & & & & & $L(17)$ & & & & \multirow{5}{*}{$\begin{array}{l}\text { MUSKRAT } \\
\text { NORWAY RAT } \\
\text { HOUSE MOUSE } \\
\text { PORCUPINE } \\
\text { ELK }\end{array}$} \\
\hline & & & & & & * & & & & \\
\hline & & & & & & 1 & & & & \\
\hline & * & & & 2 & & * & & & 1 & \\
\hline & & & & & 2 & & & & & \\
\hline & & & & 9 & & & & & & \multirow{4}{*}{$\begin{array}{l}\text { MULE DEER } \\
\text { WHITE-TAILED DEER } \\
\text { DEER sPECIES } \\
\text { MOOSE } \\
\text { PRONGHORN }\end{array}$} \\
\hline \multirow[t]{3}{*}{ *(4) } & *(10) & 3 & 2 & 6 & $D(I)$ & 12 & & & $\star(20)$ & \\
\hline & & & & & & & $\star(1)$ & & & \\
\hline & & & & & & & & & & \\
\hline 3 & 7 & 4 & 8 & 9 & 9 & 17 & 9 & 4 & 11 & TOTAL SPECIES \\
\hline
\end{tabular}


Table 1-6. SASKATCHEWAN CHRISTMAS MAMMAL COUNTS

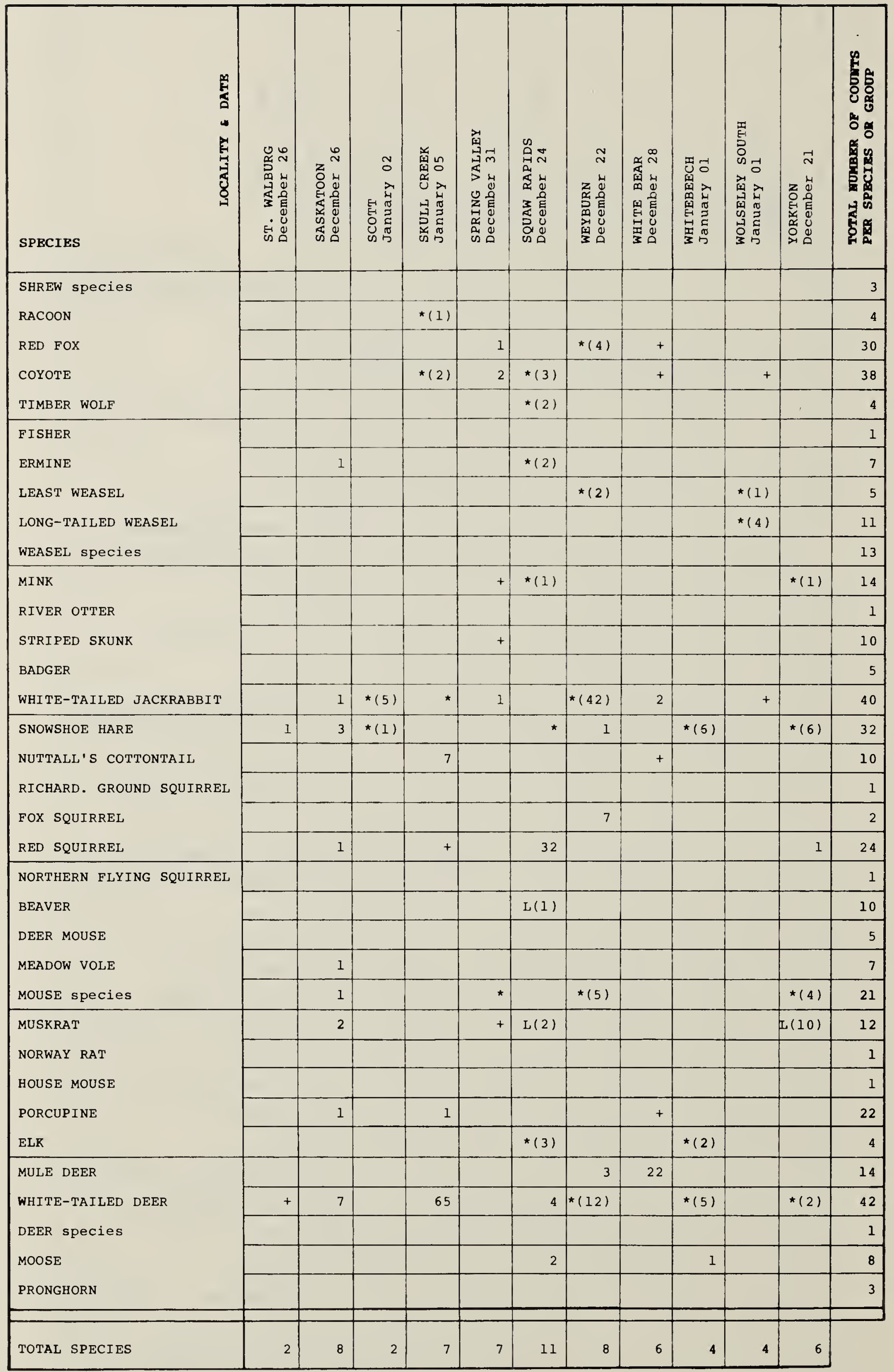

